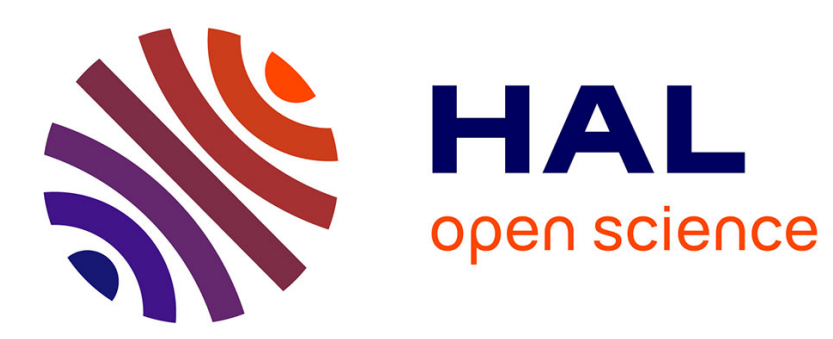

\title{
Epitaxially stabilized TiN/(Ti,Fe,Co)N multilayer thin films in (pseudo-)fcc crystal structure by sequential magnetron sputter deposition
}

C Klever, K Seemann, M Stüber, S Ulrich, H Brunken, A Ludwig, H Leiste

\section{- To cite this version:}

C Klever, K Seemann, M Stüber, S Ulrich, H Brunken, et al.. Epitaxially stabilized TiN/(Ti,Fe,Co)N multilayer thin films in (pseudo-)fcc crystal structure by sequential magnetron sputter deposition. Journal of Physics D: Applied Physics, 2010, 43 (39), pp.395406. 10.1088/0022-3727/43/39/395406 . hal-00629976

\section{HAL Id: hal-00629976 https://hal.science/hal-00629976}

Submitted on 7 Oct 2011

HAL is a multi-disciplinary open access archive for the deposit and dissemination of scientific research documents, whether they are published or not. The documents may come from teaching and research institutions in France or abroad, or from public or private research centers.
L'archive ouverte pluridisciplinaire HAL, est destinée au dépôt et à la diffusion de documents scientifiques de niveau recherche, publiés ou non, émanant des établissements d'enseignement et de recherche français ou étrangers, des laboratoires publics ou privés. 


\title{
Epitaxially stabilised TiN/(Ti,Fe,Co)N multilayer thin films in pseudo-fcc crystal structure by sequential magnetron sputter deposition
}

\author{
C Klever ${ }^{1}$, K Seemann ${ }^{1}$, M Stüber ${ }^{1}$, S Ulrich ${ }^{1}, \mathbf{H}$ \\ Brunken $^{2}$, A Ludwig ${ }^{2}$ and $\mathbf{H}$ Leiste $^{1}$ \\ ${ }^{1}$ Karlsruhe Institute of Technology (KIT), Institute for Materials Research I, \\ Hermann-von-Helmholtz-Platz 1, 76344 Eggenstein-Leopoldshafen, Germany \\ ${ }^{2}$ Ruhr-University Bochum, Institute of Materials, Universitätsstr. 150, 44780 \\ Bochum, Germany \\ E-mail: christian.klever@kit.edu
}

\begin{abstract}
Multilayer thin films were grown by non-reactive sequential magnetron sputter deposition from ceramic TiN and metallic FeCo targets addressing a combination of wear resistance and sensoric functionality. Coatings with bilayer period values ranging from $449 \mathrm{~nm}$ down to $2.6 \mathrm{~nm}$ were grown with the total amount of either material maintained constant. The multilayer thin films were post-annealed ex-situ at $600{ }^{\circ} \mathrm{C}$ for $60 \mathrm{~min}$ in vacuum.

$\mathrm{X}$-ray diffraction results imply the multilayer thin films to undergo significant changes in its crystalline structure when the bilayer period is decreased. By using high resolution transmission electron microscopy as well as selected area electron diffraction it is shown that in case of multilayer thin films with bilayer periods of several ten nanometers and higher FeCo layers and TiN layers in their respective common $\mathrm{CsCl}$ and $\mathrm{NaCl}$ type crystal structures alternate. In contrast, in the multilayer thin films with bilayer periods of only a few nanometers, grain growth across the interfaces between the individual layers takes place and a strongly textured microstructure is formed which features columns in pseudo-fcc crystal structure grown in heteroeptaxial growth mode.

It is suggested that the experimental findings imply the latter multilayer thin films to be alternately composed of TiN layers and $(\mathrm{Ti}, \mathrm{Fe}, \mathrm{Co}) \mathrm{N}$ solid solution layers which have been formed by solid state reaction during the deposition process. As the consequence, epitaxially stabilised columnar grains in strongly textured pseudo-fcc structure are formed. This structure is preserved after the annealing procedure which qualifies these coatings for use in applications where temperatures of up to $600{ }^{\circ} \mathrm{C}$ are reached.
\end{abstract}




\section{Introduction}

Wear protection coatings produced by physical vapour deposition (PVD) are used in many engineering applications since the early 1980ies. Soon after the first commercial application of single layer hard coatings for enhanced wear protection it was recognised that appropriately selected thin film materials can be combined as a multilayer system, leading to coating properties and performances significantly exceeding those of either constituent. Since then, the systematic selection of material combinations, the specific design of the multilayer architecture (e.g. individual layer thickness, interface constitution), the understanding of growth conditions, and the correlation between microstructure and properties are subject to intensive research in the field of multilayer wear resistant thin films $[1,2,3,4]$. Beyond the approach of improving the coating performance with respect to their passive functionality, studies were published focussing on the design of films which would be able to adapt their actual functionality in response to external influences as, for instance, environmental changes or mechanical load. A particular example for this type of coatings are the so-called "chameleon coatings" $[5,6,7]$. However, in-situ condition monitoring is - in general-not possible. There are hence some studies available presenting coating concepts addressing the integration of sensoric functionality into passive functional coatings. For instance, results on protective and thermal barrier coatings with integrated photoluminiscent phases were reported $[8,9]$.

In the context of the combination of passive and active functionality with the focus on use in sensor applications, we had recently published an article on the integration of a magnetic sensor phase into the standard wear resistance material TiN [10]. It had been shown that the growth of multilayer thin films by alternate deposition of TiN and FeCo layers, using non-reactive sequential magnetron sputter deposition followed by an ex-situ annealing procedure under vacuum conditions $\left(60 \mathrm{~min}, 600^{\circ} \mathrm{C}\right.$, static magnetic field with $\mu_{0} H=50 \mathrm{mT}$ ), is an appropriate technique to fabricate coatings which simultaneously can provide protective and sensoric properties (the latter through the ferromagnetic properties of $\mathrm{FeCo}$ ). It had in particular been found that by decreasing the bilayer period down to only a few nanometers important macroscopic physical properties (nanoindentation hardness, ferromagnetic softness, response to an external electromagnetic HF-field) can be improved significantly. Concerning the structure of the multilayer thin films, Low-angle X-ray diffraction (LA-XRD) and Auger electron spectroscopy (AES) depth profiles had clearly indicated that the layered structure of all films have remained unchanged after the annealing procedure [10].

This article presents a detailed microstructural analysis of these multilayer coatings. X-ray diffraction (XRD) spectra of the films in the as-deposited and the annealed state were recorded and analysed. In addition, cross-sectional transmission electron microscopy (XTEM and HRTEM) images as well as selected area electron diffraction (SAED) patterns of multilayer thin films representative for each small and high bilayer periods were recorded and evaluated. The results are discussed and a model is proposed for the specific multilayer thin film microstructure evolved at small bilayer periods as well as its formation.

\section{Experimental details}

Multilayer thin films were deposited by non-reactive sequential magnetron sputtering in a Leybold Z550 laboratory device by using FeCo and TiN targets (both with $99.9 \%$ 
nominal purity and $150 \mathrm{~mm}$ diameter $)$. Thermally oxidised Si substrates $\left(10 \times 10 \mathrm{~mm}^{2}\right.$ surface area) were placed $50 \mathrm{~mm}$ below the target center on a rotatable substrate holder and kept at floating potential during all processes. The base pressure in the chamber was $<1 \times 10^{-6}$ mbar. After sputter cleaning of targets and substrates (for a more detailed description see [10]), the films were deposited at $0.2 \mathrm{~Pa}$ working gas pressure in a Ar atmosphere (99.9999\% purity): The plasmas under both targets were run simultaneously during the film growth process; the TiN target was supplied with $700 \mathrm{~W}$ DC power whereas the FeCo target was supplied with $250 \mathrm{~W}$ HF power for the deposition of all multilayer thin films. The resulting cathode voltage (target self-bias) was $-564 \mathrm{~V}$ and $-1440 \mathrm{~V}$, respectively. The substrates were moved in stopand-go-mode between both targets, starting and stopping with TiN for each multilayer thin film. The time of interruption of substrate rotation, respectively the duration of deposition in front of the target without substrate movement, was equal below each target in order to keep the thickness ratio of the individual layers constant (neglecting deviations between the deposition rate profiles alongside the target diameters). The thickness ratio of the individual layers can hence be estimated by using the respective deposition rates $\left(D_{\mathrm{TiN}} \sim 0.93 \mathrm{~nm} \mathrm{~s}^{-1}, D_{\mathrm{FeCo}} \sim 0.33 \mathrm{~nm} \mathrm{~s}^{-1}\right.$, ascertained by means of contact profilometry). The holding time was reduced from $807 \mathrm{~s}$ to $0 \mathrm{~s}$ (the latter corresponding to the continuous rotation of the substrate holder). Accordingly, the number of bilayers was increased from $n=1$ to $n=390$ such that all films were grown with a nominal total thickness of $1 \mu \mathrm{m}$. In addition, TiN and FeCo single layer films with $1 \mu \mathrm{m}$ thickness were grown using the same deposition conditions as a reference. All multilayer thin films were post-annealed ex-situ at $600{ }^{\circ} \mathrm{C}$ for $60 \mathrm{~min}$ in vacuum $\left(p<1 \times 10^{-8}\right.$ mbar $)$ subsequent to the deposition.

The microstructure of the multilayer thin films was analysed before and after annealing by using X-ray diffraction in Bragg-Brentano geometry (XRD). Low-angle $\mathrm{X}$-ray diffraction (LA-XRD) was used in order to determine the bilayer periods lower than $10 \mathrm{~nm}$. $\mathrm{Cu} \mathrm{K} \alpha$ radiation was used.

Cross-sections of representative films were prepared for TEM analysis both in the as-deposited state and after annealing by focused ion beam cutting (FEI Quanta 3D 200i). XTEM and HRTEM images, as well as SAED patterns, were recorded from the multilayer thin film cross-sections at $200 \mathrm{kV}$ using a Tecnai F20 instrument.

\section{Results}

In figure 1, XRD patterns of the as-deposited multilayer thin films with different bilayer periods are shown. The bilayer periods higher than $10 \mathrm{~nm}$ were determined by using the deposition rates and the deposition time, the other values had been determined experimentally by means of Low-angle X-ray diffraction (LA-XRD). The diffraction patterns of $1 \mu \mathrm{m}$ thick TiN and FeCo single layers grown at identical conditions are shown as a reference. The full width at half maximum (FWHM) values of the $\mathrm{TiN}(200)$ and the TiN(111) peaks are comparatively large indicating the TiN single layer to be nanocrystalline. In particular, no amorphous regions are obtained in any of the TiN films in all cases. A dependency of the multilayer thin films' microstructure on the bilayer period can be observed: The diffraction pattern obtained from the film with $\Lambda=449 \mathrm{~nm}$ exhibits the (111) and (200) reflections of TiN in the $\mathrm{NaCl}$ tape crystal structure as well as the (110) reflection of FeCo in the CsCl type crystal structure which are the stable phases of the respective binary material at standard conditions [11]. When the bilayer period is reduced to values below $449 \mathrm{~nm}$ the $\mathrm{FeCo}(110)$ reflection 


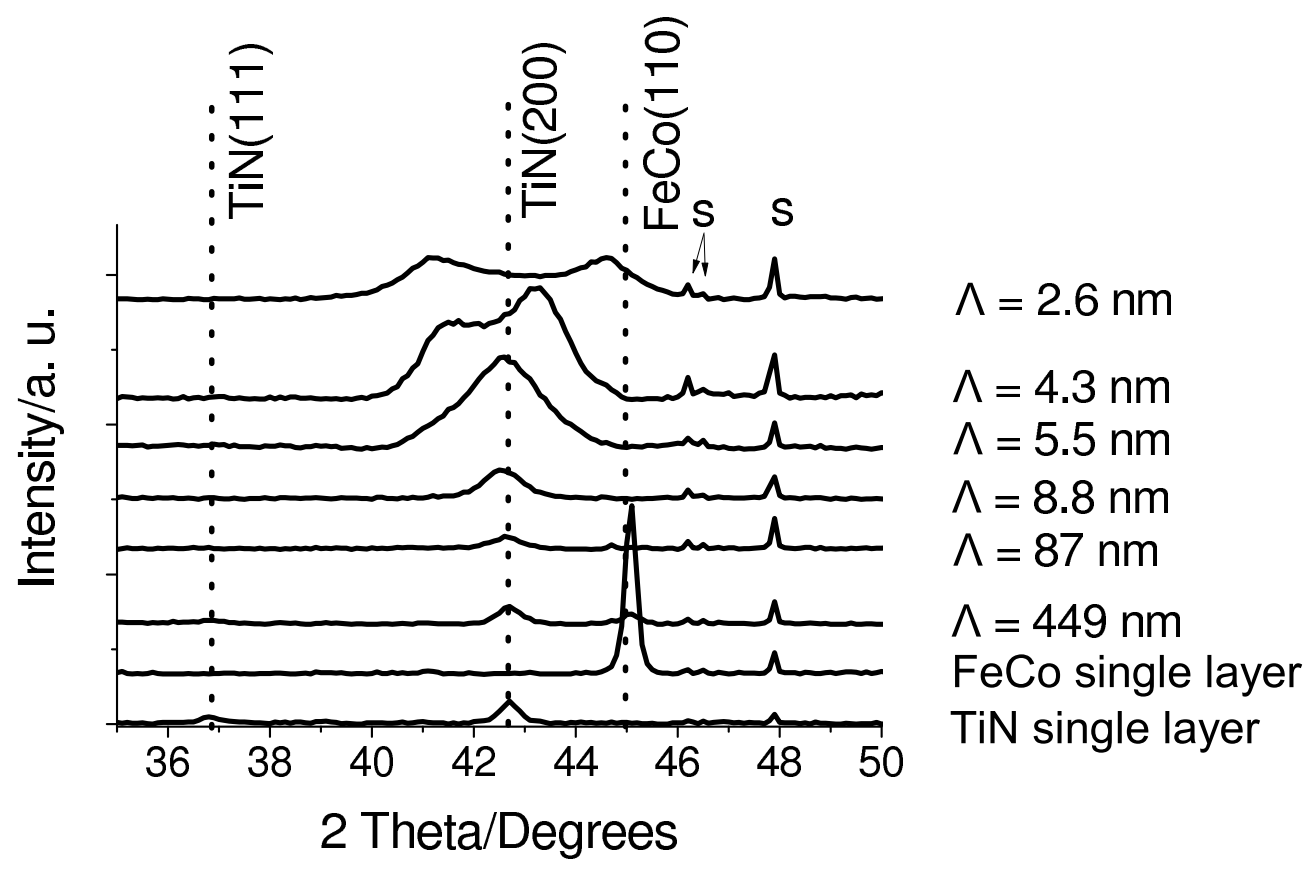

Figure 1. XRD patterns of the as-deposited multilayer thin films with different bilayer periods. The spectra of $\sim 1 \mu \mathrm{m}$ thick FeCo and TiN single layer films grown at identical deposition conditions as the multilayer thin films are shown for comparison. The dotted lines indicate the positions of the (111) and (200) reflections of $\mathrm{TiN}$ in $\mathrm{NaCl}$ type crystal structure and the (110) reflection of FeCo in $\mathrm{CsCl}$ type crystal structure. Substrate peaks are designated by 's'.

vanishes which indicates a decrease in the grain size in the FeCo phase. Down to the bilayer period of $5.5 \mathrm{~nm}$ the early disappearance of the $\mathrm{TiN}(111)$ reflection as well as a significant increase in the FWHM of the $\mathrm{TiN}(200)$ reflection imply the presence of smaller grains as a consequence of the reiterated interruption of the individual layer growth. In the diffraction patterns for multilayer thin films with bilayer periods of $4.3 \mathrm{~nm}$ and lower a striking change in the XRD patterns is observed: In the pattern of the multilayer thin film with $\Lambda=4.3 \mathrm{~nm}$, the $\operatorname{TiN}(200)$ reflection is splitted into a double-peak structure. In the multilayer thin film with the bilayer period of $2.6 \mathrm{~nm}$ the same phenomenon is observed with an even more increased distance between the two reflections.

In figure 2 a representative cross-sectional HRTEM image from the as-deposited multilayer thin film with a bilayer period of $87 \mathrm{~nm}$ is shown. The darker region on the left hand side is the FeCo layer which is covered by a TiN layer. Both films are polycrystalline and several grains obtaining distinct orientations can be observed. Both layers are separated by a predominantly incoherent interface region characterised by a high degree of lattice distortions. As is verified by the SAED pattern (shown in the inset), both TiN and FeCo are polycrystalline in its respective equilibrium crystal structure ( $\mathrm{NaCl}$ and $\mathrm{CsCl}$ type, respectively), as all diffraction rings can unambiguously allocated to the respective phase (which is explicitely shown for the five innermost diffraction rings). This result complements the findings from the the corresponding XRD pattern, the TiN(111) and the FeCo(110) peaks are not resolved 

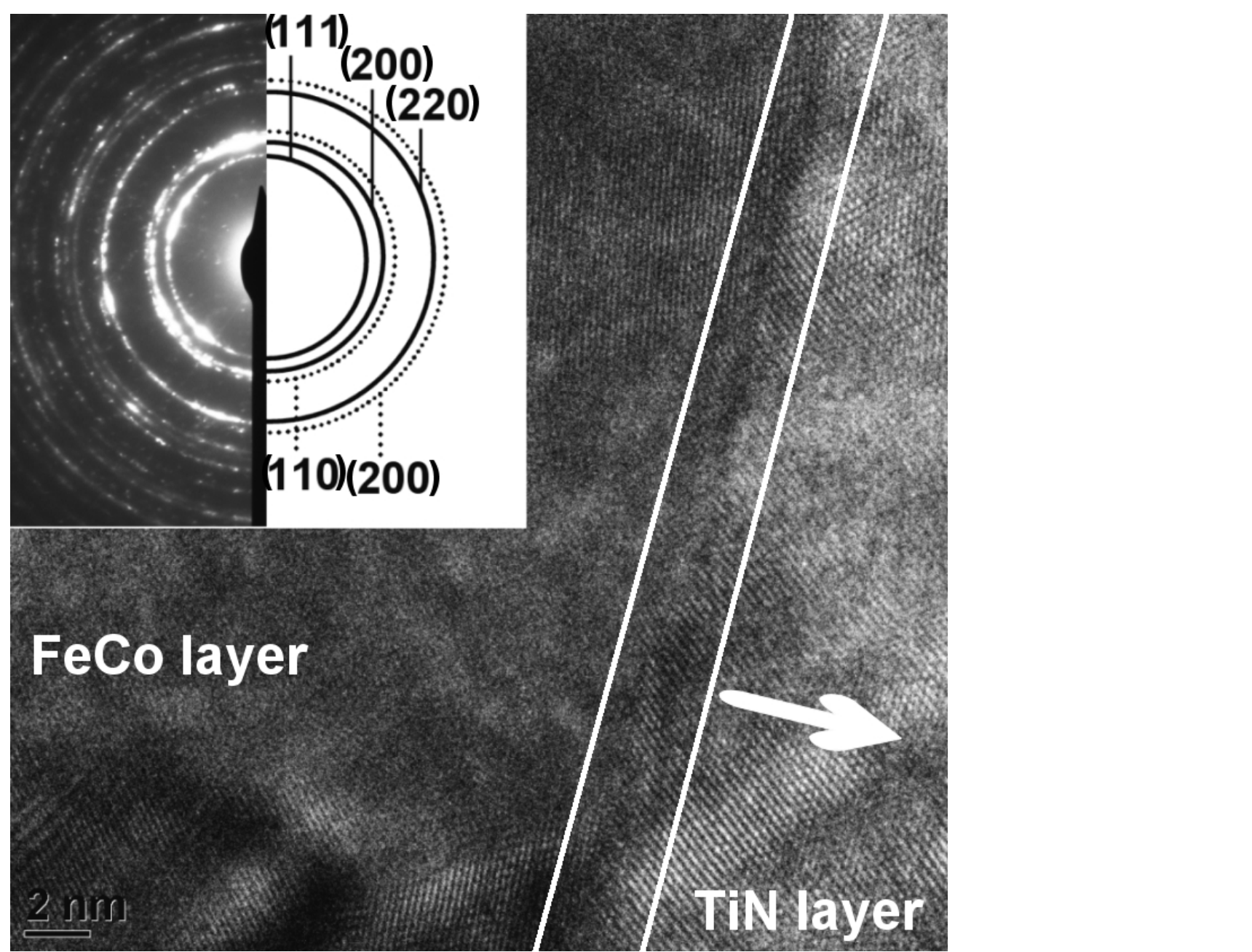

Figure 2. HRTEM image taken from the as-deposited multilayer thin film with a bilayer period of $87 \mathrm{~nm}$. The growth direction is indicated by the arrow. The interface region is adumbrated by the lines. In the inset the SAED pattern with diffraction along virtually the whole specimen cross-section is shown. The indexes of the five innermost rings are indicated; continuous rings are assigned to TiN in $\mathrm{NaCl}$ type structure, the dotted rings are assigned to FeCo in $\mathrm{CsCl}$ type structure.

in, anymore, due to the comparatively small average grain size.

The XTEM image shown in figure 3 confirms that even the multilayer thin film with the smallest bilayer period of $\Lambda=2.6 \mathrm{~nm}$ exhibits a multilayer structure, as was also been shown for the annealed film and had been supported by XRR and AES depth profiles which had been shown in an earlier publication [10]. A representative section of an HRTEM image taken from the very same film as in figure 3 is shown in figure 4 . In the detail shown, two distinct grains can be discerned which vary in the lattice orientation with respect to the electron beam direction. While the individual layers cannot be discerned due to the particular contrast conditions, pseudo-epitaxial growth along the growth direction can be well identified. The grain boundary between 


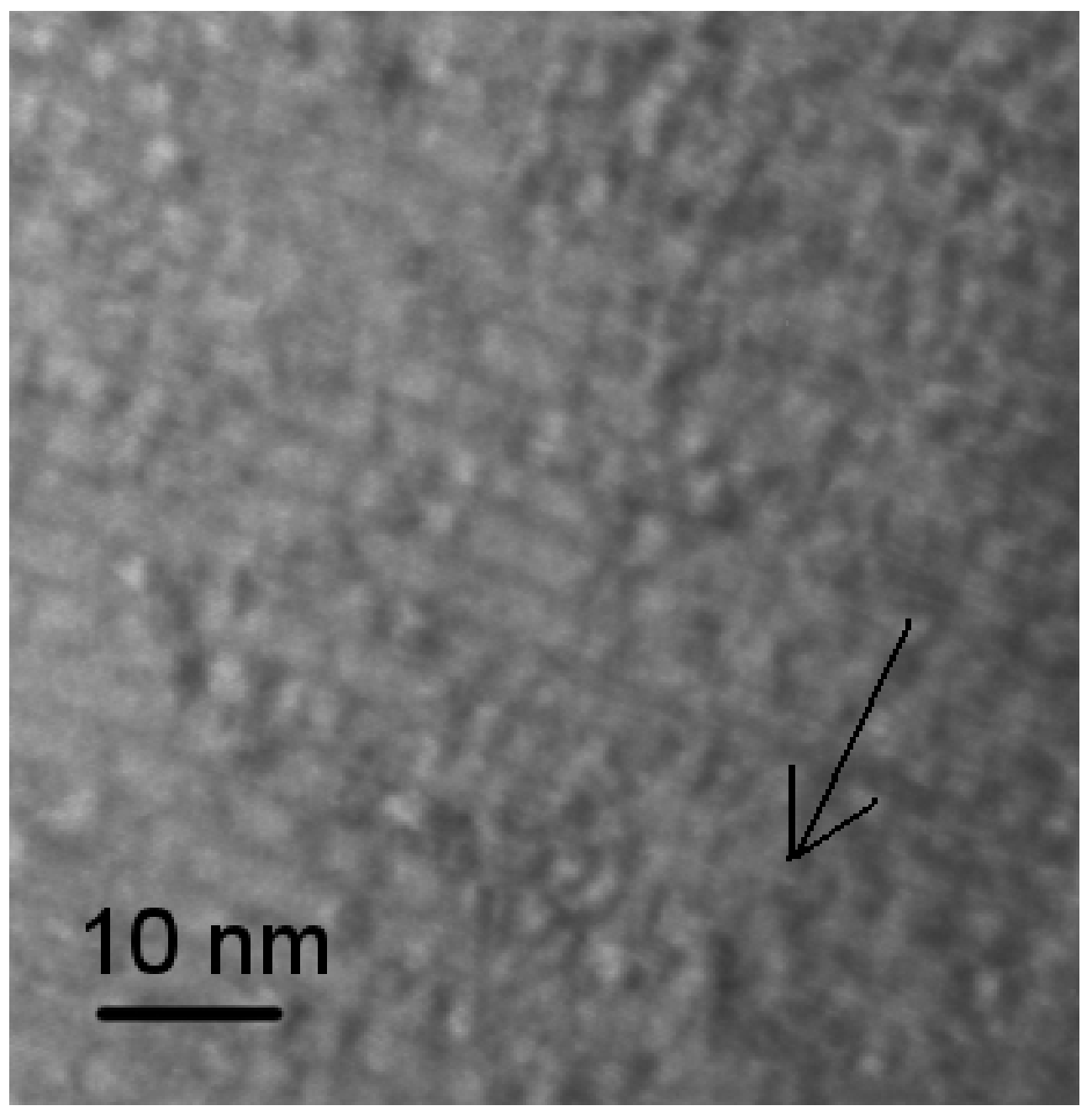

Figure 3. XTEM image taken from the as-deposited multilayer thin film with a bilayer period of $2.6 \mathrm{~nm}$. The growth direction is indicated by the arrow.

the columnar grains shown is partially coherent. In particular, the (HR)TEM images confirm that the film has grown in columnar growth mode with the lattice planes being continued across the layer interfaces. The lattice distances were determined by measuring the distance along a number of lattice planes. The values yielded are identical to those expected for $\mathrm{TiN}$ in $\mathrm{NaCl}$ type crystal structure. Based on this matter on fact the FFT insets are indexed in order to facilitate a comparison with the according SAED patterns later on.

The XRD patterns of the annealed multilayer thin films are shown in figure 5 . In the multilayer thin films with bilayer periods $\geq 5.5 \mathrm{~nm}$ a decrease in the FWHMs compared to the corresponding as-deposited state pattern implies a grain size increase during the annealing procedure both in the FeCo and the TiN phase. In the films with $\Lambda<5.5 \mathrm{~nm}$, the "peak splitting" phenomenon is likewise present after annealing albeit the reflections are shifted with respect to their previous positions. 


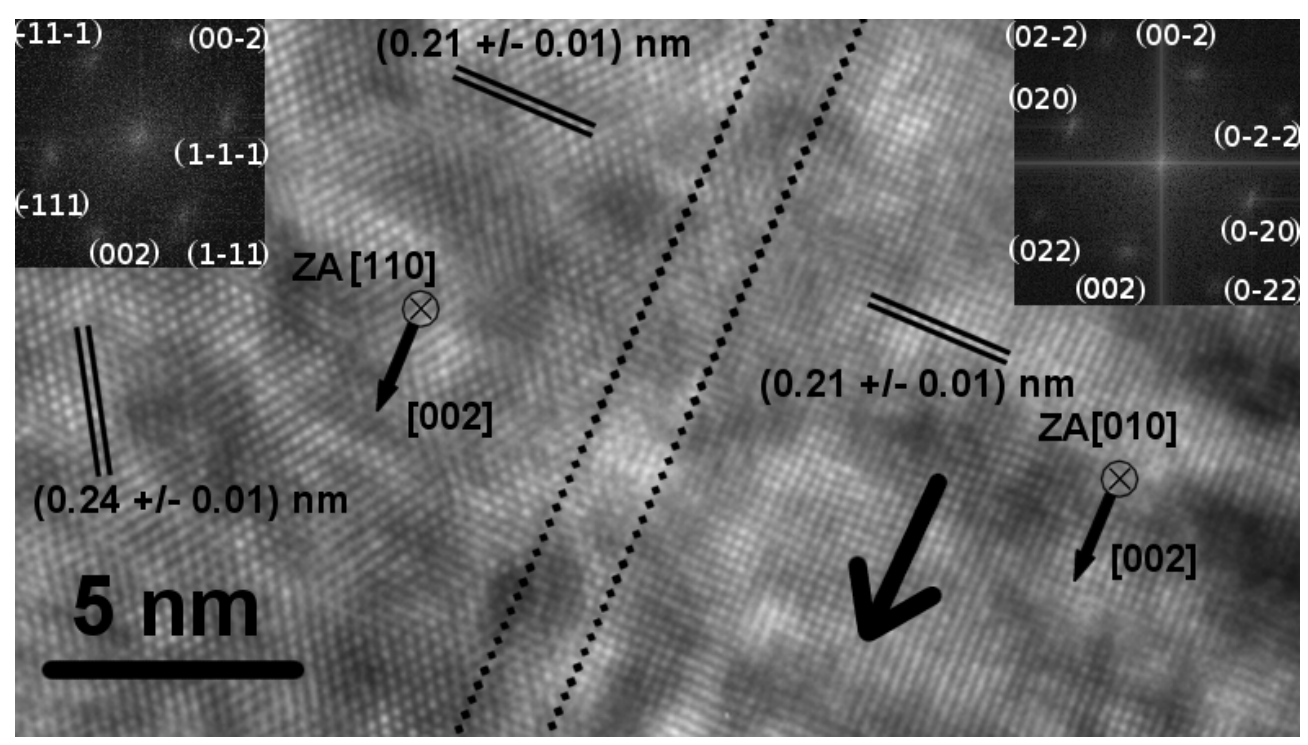

Figure 4. HRTEM image taken from the as-deposited multilayer thin film with a bilayer period of $2.6 \mathrm{~nm}$. Two distinct grains can be discerned in this section. The grain boundary region is adumbrated by the dotted lines. The respective FFT images of both grains are shown in the insets in order to facilitate the allocation to the respective diffraction patterns. The growth direction of the multilayer thin film is indicated by the arrow.

In figure 6, the SAED pattern of the multilayer thin film with the smallest bilayer period of $\Lambda=2.6 \mathrm{~nm}$ after annealing is shown. During the recording of the SAED pattern, the electron optics was adjusted such that the complete film thickness was used for diffraction processes. The sole appearance of diffraction spots without any additional ring patterns as well as the axial symmetry of the spot pattern imply the predominance of regions with pronounced texture. The SAED spots are schematically depicted in the inset in the figure: The location of all diffraction spots can be consistently indexed in terms of the superposition of diffraction patterns of a crystal in pseudo-fcc structure in consideration of distinct zone axises (ZA's). With the convention of [002] being the growth direction, the diffraction spots can be indexed as shown in figure 6 :

- Spots originated by diffraction with [010] being the ZA depicted by the black filled bulls,

- spots originated by diffraction with [110] being the ZA depicted by the bluerimmed, empty bulls,

- spots originated by diffraction with [210] being the ZA depicted by the red-rimmed squares,

- spots originated by diffraction with [310] being the ZA depicted by the green filled squares.

Proceeding the above systematics, all appearing diffraction spots can be indexed. There are explicitely no diffraction spots present indicative for crystalline regions in pseudo-bcc (or $\mathrm{CsCl}$ ) crystal structure. 


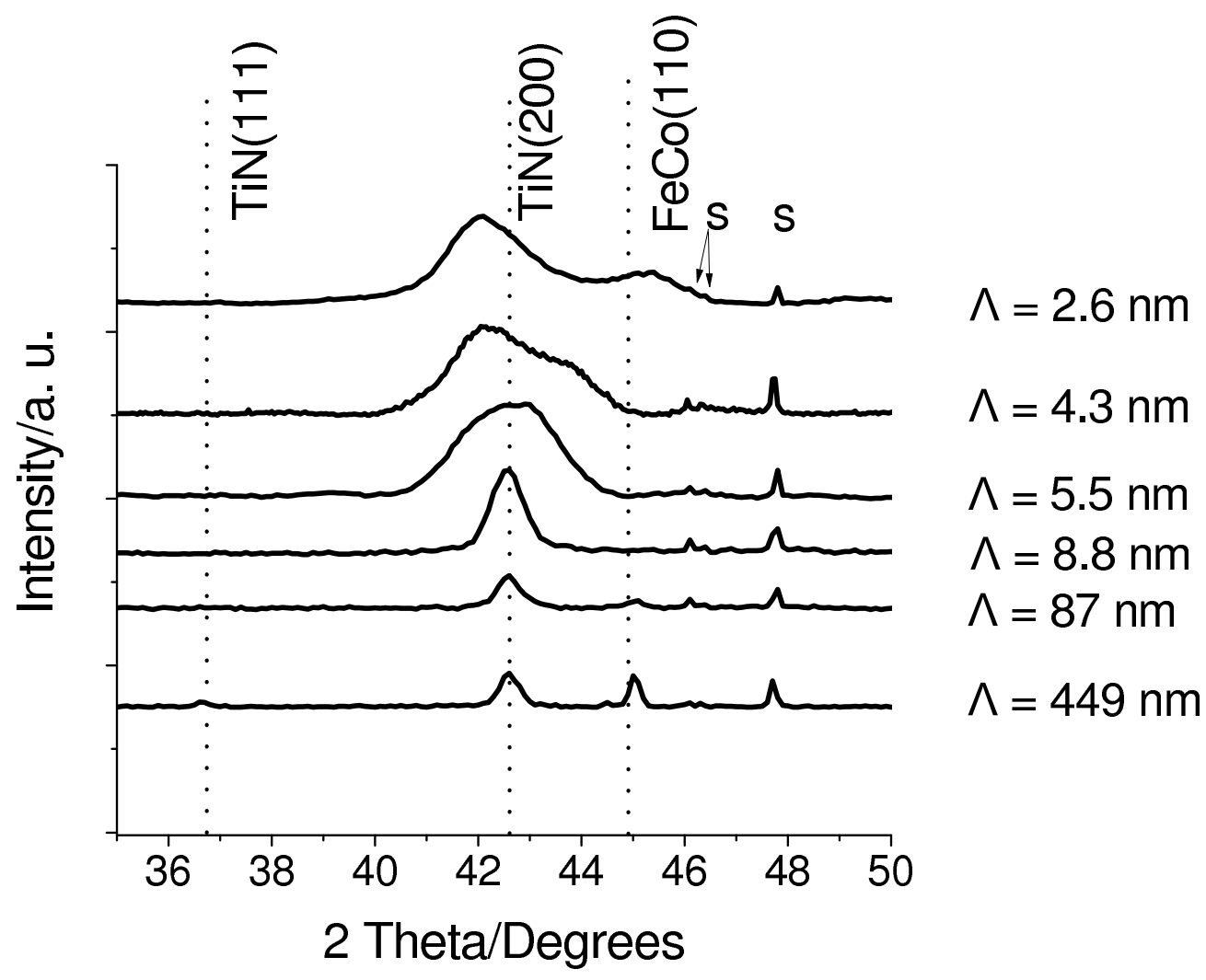

Figure 5. XRD patterns of the annealed multilayer thin films with different bilayer periods. The dotted lines indicate the positions of the (111) and (200) reflections of $\mathrm{TiN}$ in $\mathrm{NaCl}$ type crystal structure and the (110) reflection of $\mathrm{FeCo}$ in $\mathrm{CsCl}$ type crystal structure. Substrate peaks are designated by 's'.

The superlattice reflections visible at the lowest order spots with [110] being the ZA corrobate the finding that a well defined multilayer structure with bilayers of equal bilayer period is maintained after annealing. At the other diffraction spots, the multilayer reflections are not visible or considerably blurry which might be caused by the respective beam orientation. The double peak structure of the (200) reflection in the XRD pattern is not identifiable in the SAED pattern since the (200) spot is represented in maximum intensity on the negative.

\section{Discussion}

The passing of the $\operatorname{TiN}(200)$ reflection into a double peak structure in the XRD patterns when the bilayer periods are decreased implies significant microstructural changes in the multilayer thin films examined. By analysing HRTEM images and SAED patterns it is demonstrated that for small bilayer periods the multilayer thin films are predominantly grown in columnar growth mode beyond all layer interfaces with the distinct columns being nanostabilised due to pseudo-epitaxial growth in pseudo-fcc crystal structure. This behaviour is in contrast to the film growth mode for higher bilayer periods at which TiN and FeCo layers in its regular stable $\mathrm{NaCl}$ and 


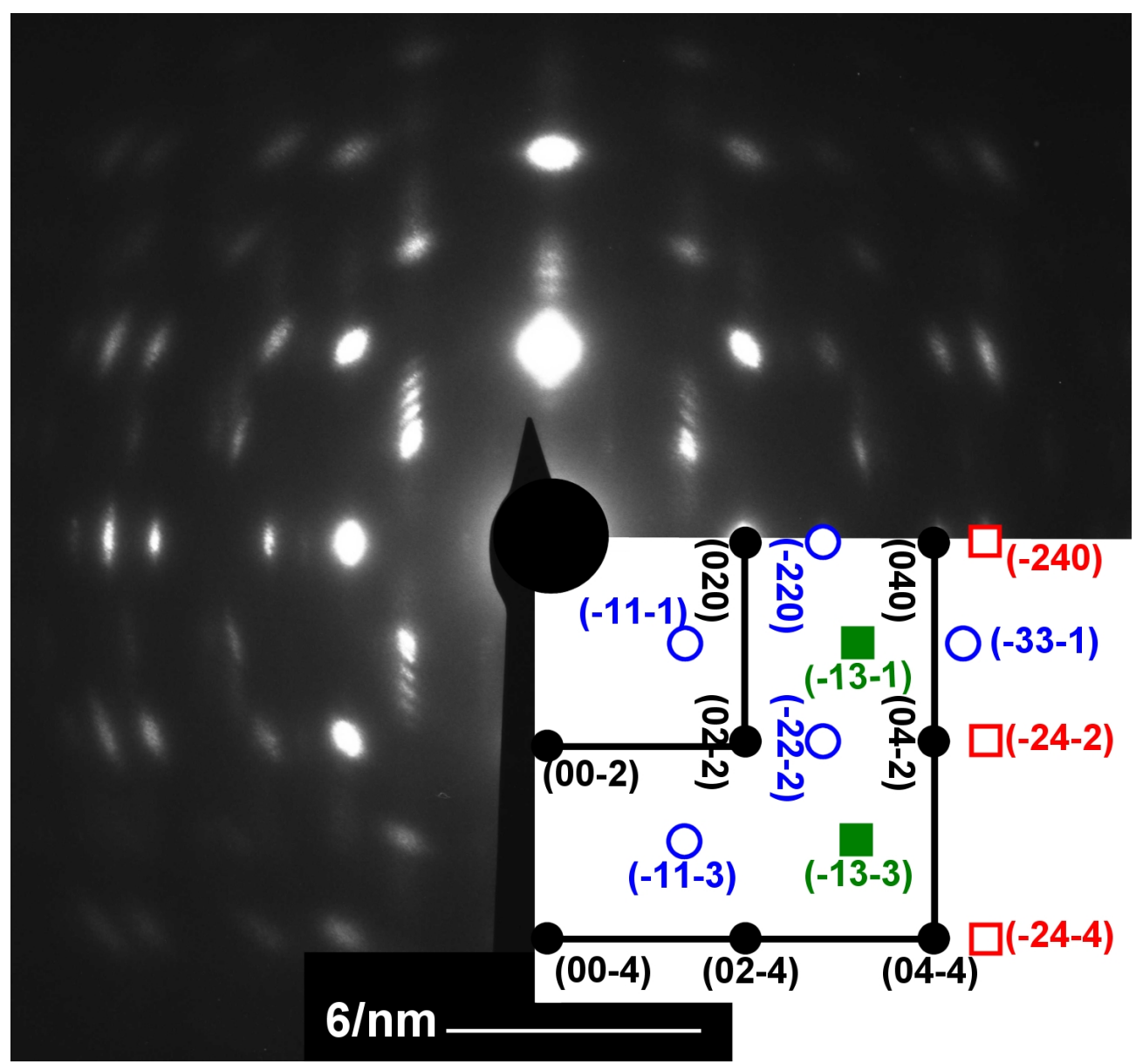

Figure 6. SAED pattern of the multilayer thin film with a bilayer period of $2.6 \mathrm{~nm}$ subsequent to $60 \mathrm{~min}$ annealing in vacuum at $600{ }^{\circ} \mathrm{C}$. The diffraction spots are schematically shown in the inset and indexed as explained in the text.

CsCl type crystal structure, respectively, alternate as expected.

It is known that $\mathrm{Fe}_{x} \mathrm{Co}_{1-x}$ can be stabilised in (pseudo-)fcc crystal structure at room temperature under appropriate conditions (choice of substrate, growth conditions, film thickness). These observations were frequently made at distinct dimensionalities as pseudomorphically grown single layer films [12, 13], multilayer thin films [14] as well as nanocrystalline films [15]. However, the lattice constants of equiatomic (pseudo-)fcc FeCo $\left(a_{\mathrm{FeCo}}=0.354 \mathrm{~nm}[11]\right)$ and TiN $\left(a_{\mathrm{TiN}}=0.424 \mathrm{~nm}[16]\right)$ would yield a relative lattice mismatch of $\left(\left(a_{\mathrm{TiN}}-a_{\mathrm{FeCo}}\right) / a_{\mathrm{TiN}}=0.165\right)$. Due to this considerable lattice mismatch pseudomorphic growth of (pseudo-)fcc FeCo layers onto $\mathrm{TiN}$ in $\mathrm{NaCl}$ type crystal structure is not expected to be possible.

By using PVD techniques thin films are grown far from thermodynamical equilibrium. As a consequence, films can be grown in crystal structures which differ from the predictions in the according (equilibrium) phase diagrams. As a prominent example, nonstoichiometric $\mathrm{TiN}_{1-x}$ films can be grown with $x$ reaching considerable (positive and negative) values while otherwise maintaining its structure which at 
standard conditions is not predicted by the according phase diagram [17]. Both the phase diagrams of the Fe-N system [18] and of the Co-N system [19] predict the existence of nitrogen-poor phases in (pseudo-)fcc crystal structure. Both the TiN and the FeCo layers were observed to not consist any amorphous regions or porosity such that interdiffusion caused by any of these facts can be excluded. Given a sufficient kinetic energy of the impinging atoms during the deposition process, the formation of a complex nitride formed from $\mathrm{Ti}, \mathrm{Fe}$, and Co should hence be possible. As a similar example, the growth of single layer $(\mathrm{Ti}, \mathrm{Fe}) \mathrm{N}$ films with $\sim 13 \%$ of $\mathrm{Ti}$ atoms being replaced by Fe atoms within a pseudo-fcc crystal structure was reported by Kirsten et al [20]. However, the films prepared by Kirsten et al were grown in a one-step process by reactive magnetron sputter deposition from a $\mathrm{Ti}$ metal target equipped with $\mathrm{Fe}$ metal pins rather than non-reactive sequential deposition from two distinct sources.

The formation of a complex nitride is the process proposed to take place in our deposition process due to the high target self bias voltage at the FeCo target and the comparatively low working gas pressure. The structural reordering process necessary for the evolution of the multilayer thin film structure presented in this study for small bilayer periods is more probable to take place during the deposition step below the FeCo target rather than that below the TiN target due to several reasons: First, the target bias voltage at the FeCo target has been considerably higher than that at the TiN target. Second, the higher mass of Fe and Co atoms compared to that of $\mathrm{Ti}$ and $\mathrm{N}$ atoms is expected to result in a higher momentum transfer during the $\mathrm{Fe}$ and Co particle impact on the substrate surface than during the Ti and $\mathrm{N}$ particle impact. The microstructure observed for small bilayer periods is thus suggested to be a consequence of collision-induced atomic mixing caused by momentum transfer subsequent to the arrival of the Fe and Co atoms at the previously deposited TiN layer.

According to the above considerations, the following structure formation model is proposed for the multilayer thin films grown with sufficiently small bilayer period:

- A (nearly) stoichiometric TiN film is grown.

- Fe and Co particles impinge. Their kinetic energy is sufficiently high in order to be built into the TiN lattice. Under the condition that the FeCo layer thickness $t_{\mathrm{FeCo}}$ remains smaller than a critical thickness $t_{\text {crit }}$, an understoichiometric $(\mathrm{Ti}, \mathrm{Fe}, \mathrm{Co}) \mathrm{N}$ solid solution with the maintained pseudo-fcc crystal structure is formed.

- The successional (nearly) stoichiometric TiN film is grown on top of the solid solution (Ti,Fe,Co)N layer. Due to $t<t_{\text {crit }}$ the epitaxial stabilisation of the solid solution layer in an fcc pseudo-structure is energetically favourable.

The formation of this TiN/(Ti,Fe,Co)N multilayer thin film in pseudo-fcc crystal structure is expected to evolve under the given experimental conditions and in the case that the nominal FeCo layer thickness $t_{\mathrm{FeCo}}$ is smaller than a critical value $t_{\mathrm{FeCo} \text {,crit }}$. The expected and the resulting multilayer structures are illustrated in figure 7 . It is expected that diffusion of $\mathrm{N}$ atoms takes place due to the now present concentration gradient resulting in the formation of understoichiometric TiN with in turn changed lattice parameters. We suggest that the lattice distances of the two distinct materials in combination with diffraction effects due to the artificial superlattice structure, as described by Fullerton et al [21], are the reasons for the distinct changes in the XRD patterns. 


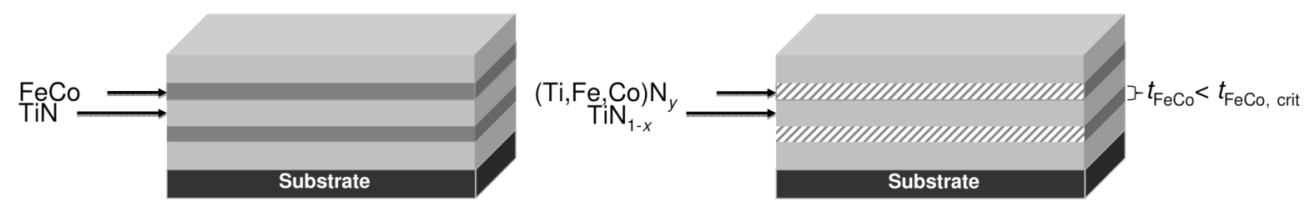

Figure 7. Illustration of the expected (left) and the resulting multilayer structure as proposed (right).

\section{Conclusions}

In the multilayer thin films investigated, a distinct change in the XRD patterns when the bilayer periods were low suggessted substantial changes in the films' microstructure. With complementary TEM experiments, the following conclusions were drawn for the multilayer thin films with small bilayer periods:

(i) As a result of appropriate multilayer thin film architecture as well as growth conditions a high volume fraction of ferromagnetic Fe and Co atoms was inserted into an fcc TiN lattice by sequential magnetron sputter deposition. A multilayer structure evolves even for bilayer periods as small as $2.6 \mathrm{~nm}$. The distinct layers grow in a pseudo-epitaxial relationship which results in the formation of columnar grains.

(ii) A $(\mathrm{Ti}, \mathrm{Fe}, \mathrm{Co}) \mathrm{N}$ solid solution in a pseudo-fcc crystal structure is suggested to be formed as a consequence of collision induced atomic mixing and subsequent nanostabilisation.

(iii) The microstructure of the multilayer thin films is thermally stable against annealing for $60 \mathrm{~min}$ at $600^{\circ} \mathrm{C}$.

The film deposition processes presented in this paper were shown to allow for the insertion of a large fraction of FeCo into an fcc crystal structure. From a technical point of view the film architecture deposited is considered to be appropriate for applications for which combined mechanical and soft magnetic properties at high temperatures (up to $600{ }^{\circ} \mathrm{C}$ ) are important.

\section{Acknowledgements}

The authors are grateful for funding by the DFG within the framework of the priority program SPP 1299 'HAUT'.

\section{References}

[1] P C Yashar and W D Sproul. Nanometer scale multilayered hard coatings. Vacuum, 55(34):179-190, 1999.

[2] P Eh Hovsepian, D B Lewis, and W-D. Münz. Recent progress in large scale manufacturing of multilayer/superlattice hard coatings. Surf. Coat. Technol., 133-134:166-175, 2000.

[3] P H Mayrhofer, C Mitterer, L Hultman, and H Clemens. Microstructural design of hard coatings. Prog. Mater. Sci., 51(8):1032-1114, 2006.

[4] M Stueber, H Holleck, H Leiste, K Seemann, S Ulrich, and C Ziebert. Concepts for the design of advanced nanoscale PVD multilayer protective thin films. J. Alloy. Compd., 483:321-333, 2008. 
[5] A A Voevodin and J S Zabinski. Supertough wear-resistant coatings with "chameleon" surface adaptation. Thin Solid Films, 370(1-2):223-231, 2000.

[6] A A Voevodin, T A Fitz, J J Hu, and J S Zabinski. Nanocomposite tribological coatings with "chameleon" surface adaptation. J. Vac. Sci. Technol. A, 20(4):1434-1444, 2002.

[7] C C Baker, J J Hu, and A A Voevodin. Preparation of $\mathrm{Al}_{2} \mathrm{O}_{3} / \mathrm{DLC} / \mathrm{Au} / \mathrm{MoS}_{2}$ chameleon coatings for space and ambient environments. Surf. Coat. Technol., 201(7):4224-4229, 2006.

[8] C Muratore, D R Clarke, J G Jones, and A A Voevodin. Smart tribological coatings with wear sensing capability. Wear, 265(5-6):913-920, 2008.

[9] R J L Steenbakker, J P Feist, R G Wellman, and J R Nicholls. Sensor thermal barrier coatings: Remote in situ condition monitoring of EB-PVD coatings at elevated temperatures. J. Eng. Gas Turb. Power, 131:041301-1-041301-9, 2009.

[10] C Klever, M Stüber, H Leiste, E Nold, K Seemann, S Ulrich, H Brunken, A Ludwig, C Thede, and E Quandt. Multifunctional FeCo/TiN multilayer thin films with combined magnetic and protective properties. Adv. Eng. Mater., 11(12):969-975, 2009.

[11] T Sourmail. Near equiatomic FeCo alloys: Constitution, mechanical and magnetic properties. Prog. Mater. Sci, 50(7):816-880, 2005.

[12] A Winkelmann, M Przybylski, F Luo, Y Shi, and J Barthel. Perpendicular magnetic anisotropy induced by tetragonal distortion of FeCo alloy films grown on $\mathrm{Pd}(001)$. Phys. Rev. Lett., 96(25), 2006.

[13] F Yildiz, M Przybylski, X-D Ma, and J Kirschner. Strong perpendicular anisotropy in $\mathrm{Fe}_{1-x} \mathrm{Co}_{x}$ alloy films epitaxially grown on mismatching $\operatorname{Pd}(001), \operatorname{Ir}(001)$, and $\operatorname{Rh}(001)$ substrates. Phys. Rev. B, 80(6), 2009.

[14] I L Graff, J Geshev, S R Teixeira, L Amaral, and A Traverse. Atomic level mixing induced by $\mathrm{Kr}$ irradiation of $\mathrm{FeCo} / \mathrm{Cu}$ multilayers. J. Appl. Phys., 103(3):033505-11, 2008.

[15] Y Liu, C Y Tan, Z W Liu, and C K Ong. FeCoSiN film with ordered FeCo nanoparticles embedded in a Si-rich matrix. Appl. Phys. Lett., 90(11):112506-3, 2007.

[16] JCPDF-file 38-1420.

[17] P E Schmid, M S Sunaga, and F Levy. Optical and electronic properties of sputtered TiN thin $_{x}$ films. J. Vac. Sci. Technol. A, 16(5):2870-2875, 1998.

[18] B Predel. Fe-N (Iron-Nitrogen), volume 5e: Dy-Er-Fr-Mo of SpringerMaterials - The LandoltBörnstein Database. Springer-Verlag, Berlin/Heidelberg, 1995.

[19] B Predel. Co-N (Cobalt-Nitrogen), volume 5e: Dy-Er-Fr-Mo of SpringerMaterials - The LandoltBörnstein Database. Springer-Verlag, Berlin/Heidelberg, 1995.

[20] A Kirsten, C Pietzsch, and H Oettel. Mössbauer investigations of (Ti,Fe)N hard coatings. Thin Solid Films, 288(1-2):198-201, 1996.

[21] E E Fullerton, I K Schuller, H Vanderstraeten, and Y Bruynseraede. Structural refinement of superlattices from x-ray diffraction. Phys. Rev. B, 45(16):9292, 1992. 